\title{
CERTAIN NONLINEAR DIFFERENTIAL POLYNOMIAL SHARING A NONZERO POLYNOMIAL IM
}

\author{
By
}

Abhijit BanerJeE and Sujoy Majumder

\begin{abstract}
We study the uniqueness of meromorphic functions when certain nonlinear differential polynomial sharing a nonzero polynomial having common poles and thus radically improve and extend some recent results due to of Wang-Lu-Chen [17], Sahoo [16] and Liu and Yang [14].
\end{abstract}

\section{Introduction, Definitions and Results}

In this paper by meromorphic functions we shall always mean meromorphic functions in the complex plane.

Let $f$ and $g$ be two non-constant meromorphic functions and let $a$ be a finite complex number. We say that $f$ and $g$ share $a \mathrm{CM}$, provided that $f-a$ and $g-a$ have the same zeros with the same multiplicities. Similarly, we say that $f$ and $g$ share $a \mathrm{IM}$, provided that $f-a$ and $g-a$ have the same zeros ignoring multiplicities. In addition we say that $f$ and $g$ share $\infty \mathrm{CM}$, if $1 / f$ and $1 / g$ share $0 \mathrm{CM}$, and we say that $f$ and $g$ share $\infty$ IM, if $1 / f$ and $1 / g$ share 0 IM.

We adopt the standard notations of value distribution theory (see [7]). We denote by $T(r)$ the maximum of $T(r, f)$ and $T(r, g)$. The notation $S(r)$ denotes any quantity satisfying $S(r)=o(T(r))$ as $r \rightarrow \infty$, outside of a possible exceptional set of finite linear measure.

Throughout this paper, we use $P(\omega)=a_{m} \omega^{m}+a_{m-1} \omega^{m-1}+\cdots+a_{1} \omega+a_{0}$ as a nonzero polynomial in $w$ with $a_{0}, a_{1}, \ldots, a_{m}$ as complex constants. We also

2010 Mathematics Subject Classification: Primary 30D35.

Key words and phrases: Uniqueness, Meromorphic function, Non-linear differential polynomials.

The first author is thankful to DST-PURSE programme for financial assistance.

Typeset by $\mathcal{A}_{\mathcal{M}} \mathcal{S}$-IATEX.

Received September 29, 2014.

Revised February 24, 2015. 
need the following definition:

$$
\Theta(a ; f)=1-\limsup _{r \rightarrow \infty} \frac{\bar{N}(r, a ; f)}{T(r, f)},
$$

where $a$ is a value in the extended complex plane.

For a positive integer $m$ and a number $\lambda$, let $m^{*}=\chi_{\lambda} m$, where $\chi_{\lambda}=0$ if $\lambda=0$ and $\chi_{\lambda}=1$ if $\lambda \neq 0$. For the sake of simplicity we also use the notation

$$
m^{* *}= \begin{cases}m, & \text { if } \operatorname{deg}(P(\omega))=m(\geq 1) \\ 0, & \text { if } P(\omega)=a_{0}\end{cases}
$$

We note that when $P(\omega)=a_{m} \omega^{m}+a_{0}$ is a non constant polynomial then $m^{* *}=m^{*}$.

In 1959, W. K. Hayman ([8] see also [7], Corollary of Theorem 9) proved the following theorem.

THEOREM A. Let $f$ be a transcendental meromorphic function and $n(\geq 3)$ is an integer. Then $f^{n} f^{\prime}=1$ has infinitely many solutions.

During the last couple of years or so, authors have given priorities to the uniqueness results, involving the concept of shared values. First it has been assumed that $f$ and $g$ are non-constant meromorphic functions in $\mathbf{C}$ and $P$ is a certain differential polynomial such that $P[f]$ and $P[g]$ share one or possibly two values. Then the question arises under which assumptions on $P$, on the sharing hypothesis of the values and others one can conclude that $f \equiv g$ or that $f$ and $g$ are closely related in some other way. In this direction we first recall the following results of Fang and Hua [4], Yang and Hua [19] who obtained a uniqueness theorem corresponding to Theorem $A$.

THEOREM B. Let $f$ and $g$ be two non-constant entire (meromorphic) functions, $n \geq 6(\geq 11)$ be a positive integer. If $f^{n} f^{\prime}$ and $g^{n} g^{\prime}$ share $1 C M$, then either $f(z)=c_{1} e^{c z}, g(z)=c_{2} e^{-c z}$, where $c_{1}, c_{2}$ and $c$ are three constants satisfying $\left(c_{1} c_{2}\right)^{n+1} c^{2}=-1$ or $f \equiv$ tg for a constant $t$ such that $t^{n+1}=1$.

Afterwards, many elegant results have been obtained by different authors in this context. Among them we mention first the following two results due to J. Wang, W. Lu and Y. Chen [17] as this will be pertinent with our future discussions. 
THEOREM C [17]. Let $f$ and $g$ be two non-constant meromorphic functions, and $n, k, m$ be three positive integers with $n>9 k+6 m^{*}+13$. Suppose $\left(f^{n}\left(\mu f^{m}+\lambda\right)\right)^{(k)},\left(g^{n}\left(\mu g^{m}+\lambda\right)\right)^{(k)}$ share $1 I M$, where $\lambda, \mu$ are constants such that $|\lambda|+|\mu| \neq 0$, and $f, g$ share $\infty I M$.

(i) If $\lambda \mu \neq 0, m>1$ and $(n, n+m)=1$, or while $m=1$ and $\Theta(\infty, f)>2 / n$, then $f \equiv g$;

(ii) if $\lambda \mu=0$, then either $f=t g$, where $t$ is a constant satisfying $t^{n+m^{*}}=1$ or $f=c_{1} e^{c z^{2}}, g=c_{2} e^{-c z^{2}}$, where $c_{1}, c_{2}$ and $c$ are three constants such that $(-1)^{k} \lambda^{2}\left(c_{1} c_{2}\right)^{n+m^{*}}\left[\left(n+m^{*}\right) c\right]^{2 k}=1$ or $(-1)^{k} \mu^{2}\left(c_{1} c_{2}\right)^{n+m^{*}}\left[\left(n+m^{*}\right) c\right]^{2 k}=1$.

THEOREM D [17]. Let $f, g$ be two transcendental meromorphic functions, and $n, k, m$ be three positive integers with $n>9 k+4 m+15$. If $\left(f^{n}(f-1)^{m}\right)^{(k)}$, $\left(g^{n}(g-1)^{m}\right)^{(k)}$ share 1 IM and $f, g$ share $\infty I M$, then either $f \equiv g$ or $f^{n}(f-1)^{m}$ $\equiv g^{n}(g-1)^{m}$.

In 2010, P. Sahoo treated the problem of more generalized differential polynomial sharing fixed point than that considered in the above two theorems.

P. Sahoo [16] obtained the following result.

THEOREM E [16]. Let $f$ and $g$ be two transcendental meromorphic functions, and let $n, k$ and $m$ be three positive integers such that $n>9 k+4 m+13$. Let $P(z)=a_{m} z^{m}+\cdots+a_{1} z+a_{0}$, where $a_{0}(\neq 0), a_{1}, \ldots, a_{m}(\neq 0)$ are complex constants. Suppose that $\left[f^{n} P(f)\right]^{(k)},\left[g^{n} P(g)\right]^{(k)}$ share $z I M$ and $f, g$ share $\infty I M$. Then either $f(z)=\operatorname{tg}(z)$ for a constant $t$ such that $t^{d}=1$, where $d=(n+m, \ldots$, $n+m-i, \ldots, n), a_{m-i} \neq 0$ for some $i=0,1,2, \ldots, m$ or $f$ and $g$ satisfy the algebraic equation $R(f, g) \equiv 0$, where

$$
R(f, g)=f^{n} P(f)-g^{n} P(g)
$$

For entire functions, sharing fixed point CM, Qi-Yang [15] and Dou-Qi-Yang [3] obtained more generalized results as follows.

THEOREM F. Let $f$ and $g$ be two transcendental entire functions, and let $n, k$ and $m$ be three positive integers with $n>2 k+m^{*}+4, \lambda, \mu$ be two constants such that $|\lambda|+|\mu| \neq 0$. If $\left[f^{n}\left(\lambda f^{m}+\mu\right)\right]^{(k)}$ and $\left[g^{n}\left(\lambda g^{m}+\mu\right)\right]^{(k)}$ share $z C M$, then one of the following conclusions hold:

(1) If $\lambda \mu \neq 0$, then $f^{d} \equiv g^{d}$, where $d=\operatorname{gcd}(n, m)$; in particular $f \equiv g$, when $d=1$; 
(2) If $\lambda \mu=0$, then $f \equiv c g$, where $c$ is a constant satisfying $c^{n+m^{*}}=1$, or $k=1$ and $f(z)=b_{1} e^{b z^{2}}, g(z)=b_{2} e^{-b z^{2}}$, for some constants $b_{1}, b_{2}$ and $b$ that satisfy $4(\lambda+\mu)^{2}\left(b_{1} b_{2}\right)^{n+m^{*}}\left[\left(n+m^{*}\right) b\right]^{2}=-1$.

THEOREM G. Let $P(\omega)=a_{m} \omega^{m}+a_{m-1} \omega^{m-1}+\cdots+a_{1} \omega+a_{0}$ or $P(\omega)=C$, where $a_{0}, a_{1}, \ldots, a_{m-1}, a_{m}(\neq 0), C(\neq 0)$ are complex constants. Suppose that $f$ and $g$ be two transcendental entire functions, and let $n, k$ and $m$ be three positive integers with $n>2 k+m^{* *}+4$. If $\left[f^{n} P(f)\right]^{(k)}$ and $\left[g^{n} P(g)\right]^{(k)}$ share $z C M$ then the following conclusions hold:

(i) If $P(w)=a_{m} w^{m}+a_{m-1} w^{m-1}+\cdots+a_{1} w+a_{0}$ is not a monomial, then $f(z) \equiv \operatorname{tg}(z)$ for a constant $t$ such that $t^{d}=1$, where $d=\operatorname{gcd}(n+m, \ldots$, $n+m-i, \ldots, n), a_{m-i} \neq 0$ for some $i=0,1,2, \ldots, m$, or $f$ and $g$ satisfy the algebraic equation $R(f, g) \equiv 0$, where $R\left(\omega_{1}, \omega_{2}\right)=\omega_{1}^{n}\left(a_{m} \omega_{1}^{m}+\right.$ $\left.a_{m-1} \omega_{1}^{m-1}+\cdots+a_{0}\right)-\omega_{2}^{n}\left(a_{m} \omega_{2}^{m}+a_{m-1} \omega_{2}^{m-1}+\cdots+a_{0}\right)$;

(ii) If $P(w) \equiv C$ or $P(z)=a_{m} z^{m}$ then $f \equiv t g$ for some constant $t$ such that $t^{n+m^{* *}}=1$, or then $f=b_{1} e^{b z^{2}}, g=b_{2} e^{-b z^{2}}$, for three constants $b_{1}, b_{2}$ and $b$ that satisfy $4 a_{m}^{2}\left(b_{1} b_{2}\right)^{n+m}[(n+m) b]^{2}=-1$ or $4 C^{2}\left(b_{1} b_{2}\right)^{n}[n b]^{2}=-1$.

Very recently, Liu and Yang [14] replaced the CM fixed point sharing concept by that of IM sharing one in the above two theorems. They proved the following results:

THEOREM H. Let $f$ and $g$ be two transcendental entire functions, and let $n, k$ and $m$ be three positive integers with $n>5 k+4 m^{*}+7, \lambda, \mu$ be two constants such that $|\lambda|+|\mu| \neq 0$. If $\left[f^{n}\left(\lambda f^{m}+\mu\right)\right]^{(k)}$ and $\left[g^{n}\left(\lambda g^{m}+\mu\right)\right]^{(k)}$ share $z$ IM, then the conclusion of Theorem $\mathrm{F}$ holds.

THEOREM I. Let $P(\omega)=a_{m} \omega^{m}+a_{m-1} \omega^{m-1}+\cdots+a_{1} \omega+a_{0}$ or $P(\omega)=C$, where $a_{0}, a_{1}, \ldots, a_{m-1}, a_{m}(\neq 0), C(\neq 0)$ are complex constants. Suppose that $f$ and $g$ be two transcendental entire functions, and let $n, k$ and $m$ be three positive integers with $n>5 k+4 m^{* *}+7$. If $\left[f^{n} P(f)\right]^{(k)}$ and $\left[g^{n} P(g)\right]^{(k)}$ share $z$ IM then the then the conclusion of Theorem $\mathrm{G}$ holds.

So it will be interesting to investigate the above theorems in case of meromorphic functions sharing a non-zero polynomial IM having common poles so that all the results can be brought under a single umbrella. The main intention of the paper is to obtain a result in a more compact and convenient way so that it will improve, generalize and extend all the previous results as far as IM sharing 
is concerned. We have also reduced the lower bound of $n$ to some extent in comparison to that obtained in Themes $C, D$ and $E$. Following two theorems are the main results of this paper:

THEOREM 1. Let $f$ and $g$ be two transcendental meromorphic functions, let $n, k, m$ be three positive integers such that $n>9 k+4 m^{*}+11$ and $\lambda, \mu$ be two constants such that $|\lambda|+|\mu| \neq 0$. Let $p(z)$ be a non zero polynomial with $\operatorname{deg}(p) \leq n-1$. Suppose $\left[f^{n}\left(\lambda f^{m}+\mu\right)\right]^{(k)}$ and $\left[g^{n}\left(\lambda g^{m}+\mu\right)\right]^{(k)}$ share $p$ IM and $f$, $g$ share $\infty I M$. Then one of the following conclusions holds:

(1) when $\lambda \mu \neq 0$, if $m \geq 2$, then $f \equiv t g$, for some constant $t$, satisfying $t^{d}=1$, where $d=\operatorname{gcd}(n, m)$; if $m=1$ and $\Theta(\infty ; f)+\Theta(\infty ; g)>\frac{4}{n}$, then $f \equiv g$;

(2) when $\lambda \mu=0$, then either $f \equiv t g$, where $t$ is a constant satisfying $t^{n+m^{*}}=1$, or if $p(z)$ is not a constant then $f=c_{1} e^{c Q(z)}, g=c_{2} e^{-c Q(z)}$, where $Q(z)=$ $\int_{0}^{z} p(z) d z$ and $c_{1}, c_{2}$ and $c$ are three constants satisfying either $\mu^{2}(n c)^{2}\left(c_{1} c_{2}\right)^{n}=-1$ or $\lambda^{2}[(n+m) c]^{2}\left(c_{1} c_{2}\right)^{n+m}=-1$,

if $p(z)$ is a nonzero constant $b$, then $f=c_{3} e^{d z}, g=c_{4} e^{-d z}$, where $c_{3}, c_{4}$ and $d$ are three constants satisfying either $(-1)^{k} \lambda^{2}\left(c_{1} c_{2}\right)^{n+m}[(n+m) c]^{2 k}=$ $b^{2}$ or $(-1)^{k} \mu^{2}\left(c_{1} c_{2}\right)^{n}[n c]^{2 k}=b^{2}$.

THEOREM 2. Let $f$ and $g$ be two transcendental meromorphic functions and $P(\omega)=a_{m} \omega^{m}+a_{m-1} \omega^{m-1}+\cdots+a_{1} \omega+a_{0}$ or $P(\omega)=C$, where $a_{0}, a_{1}, \ldots, a_{m-1}$, $a_{m}(\neq 0), C(\neq 0)$ are complex constants. Let $n, k$ and $m$ be three positive integers with $n>9 k+4 m^{* *}+11$ and $p(z)$ be a non zero polynomial with $\operatorname{deg}(p) \leq n-1$. Suppose $\left[f^{n} P(f)\right]^{(k)},\left[g^{n} P(g)\right]^{(k)}$ share $p$ IM and $f, g$ share $\infty I M$, then

(I) when $P(w)=a_{m} w^{m}+a_{m-1} w^{m-1}+\cdots+a_{1} w+a_{0}$ is a non-constant polynomial such that $P(w) \neq a_{m} w^{m}$, one of the following two cases holds:

(I1) $f(z) \equiv \operatorname{tg}(z)$ for a constant $t$ such that $t^{d}=1$, where $d=$ $\operatorname{gcd}(n+m, \ldots, n+m-i, \ldots, n), a_{m-i} \neq 0$ for some $i=1,2, \ldots, m$,

(I2) $f$ and $g$ satisfy the algebraic equation $R(f, g) \equiv 0$, where $R\left(\omega_{1}, \omega_{2}\right)=$ $\omega_{1}^{n}\left(a_{m} \omega_{1}^{m}+a_{m-1} \omega_{1}^{m-1}+\cdots+a_{0}\right)-\omega_{2}^{n}\left(a_{m} \omega_{2}^{m}+a_{m-1} \omega_{2}^{m-1}+\cdots+a_{0}\right) ;$

(II) when $P(\omega) \equiv C$ or $P(w)=a_{m} w^{m}$; one of the following two cases holds:

(II1) $f \equiv$ tg for some constant $t$ such that $t^{n+m^{* *}}=1$,

(II2) if $p(z)$ is not a constant, then $f=c_{1} e^{c Q(z)}, g=c_{2} e^{-c Q(z)}$, where $Q(z)=\int_{0}^{z} p(z) d z, \quad c_{1}, \quad c_{2}$ and $c$ are constants such that either $a_{m}^{2}\left(c_{1} c_{2}\right)^{n+m}[(n+m) c]^{2}=-1$ or $C^{2}\left(c_{1} c_{2}\right)^{n}[n c]^{2}=-1$,

if $p(z)$ is a nonzero constant $b$, then $f=c_{3} e^{c z}, g=c_{4} e^{-c z}$, where $c_{3}$, $c_{4}$ and $c$ are constants such that either $(-1)^{k} a_{m}^{2}\left(c_{3} c_{4}\right)^{n+m}[(n+m) c]^{2 k}$ $=b^{2}$ or $(-1)^{k} C^{2}\left(c_{3} c_{4}\right)^{n}[n c]^{2 k}=b^{2}$. 
REMARK 1. When $f$ and $g$ are transcendental entire functions then the conditions $n>9 k+4 m^{*}+11$ and $n>9 k+4 m^{* *}+11$ of the Theorems 1 and 2 will be replaced by respectively $n>5 k+4 m^{*}+7$ and $n>5 k+4 m^{* *}+7$.

We now explain some definitions and notations which are used in the paper.

Definition 1 [13]. Let $p$ be a positive integer and $a \in \mathbf{C} \cup\{\infty\}$.

(i) $N(r, a ; f \mid \geq p) \quad(\bar{N}(r, a ; f \mid \geq p))$ denotes the counting function (reduced counting function) of those a-points of $f$ whose multiplicities are not less than $p$.

(ii) $N(r, a ; f \mid \leq p) \quad(\bar{N}(r, a ; f \mid \leq p))$ denotes the counting function (reduced counting function) of those a-points of $f$ whose multiplicities are not greater than $p$.

Definition 2 [21]. For $a \in \mathbf{C} \cup\{\infty\}$ and a positive integer $p$ we denote by $N_{p}(r, a ; f)$ the sum $\bar{N}(r, a ; f)+\bar{N}(r, a ; f \mid \geq 2)+\cdots+\bar{N}(r, a ; f \mid \geq p)$. Clearly $N_{1}(r, a ; f)=\bar{N}(r, a ; f)$.

Definition 3. Let $a, b \in \mathbf{C} \cup\{\infty\}$. Let $p$ be a positive integer. We denote by $\bar{N}(r, a ; f|\geq p| g=b) \quad(\bar{N}(r, a ; f|\geq p| g \neq b))$ the reduced counting function of those a-points of $f$ with multiplicities $\geq p$, which are the b-points (not the b-points) of $g$.

DeFINITION $4[1,2]$. Let $f$ and $g$ be two non-constant meromorphic functions such that $f$ and $g$ share the value 1 IM. Let $z_{0}$ be a 1-point of $f$ with multiplicity $p$, a 1-point of $g$ with multiplicity $q$. We denote by $\bar{N}_{L}(r, 1 ; f)$ the counting function of those 1-points of $f$ and $g$ where $p>q$, by $N_{E}^{1)}(r, 1 ; f)$ the counting function of those 1-points of $f$ and $g$ where $p=q=1$ and by $\bar{N}_{E}^{(2}(r, 1 ; f)$ the counting function of those 1-points of $f$ and $g$ where $p=q \geq 2$, each point in these counting functions is counted only once. In the same way we can define $\bar{N}_{L}(r, 1 ; g), N_{E}^{1)}(r, 1 ; g), \bar{N}_{E}^{(2}(r, 1 ; g)$.

Definition $5[1,2]$. Let $k$ be a positive integer. Let $f$ and $g$ be two nonconstant meromorphic functions such that $f$ and $g$ share the value 1 IM. Let $z_{0}$ be a 1-point of $f$ with multiplicity $p$, a 1-point of $g$ with multiplicity $q$. We denote by $\bar{N}_{f>k}(r, 1 ; g)$ the reduced counting function of those 1-points of $f$ and $g$ such that $p>q=k . \quad \bar{N}_{g>k}(r, 1 ; f)$ is defined analogously. 
Definition 6 [9, 10]. Let $f, g$ share a value a IM. We denote by $\bar{N}_{*}(r, a ; f, g)$ the reduced counting function of those a-points of $f$ whose multiplicities differ from the multiplicities of the corresponding a-points of $g$.

Clearly

$\bar{N}_{*}(r, a ; f, g) \equiv \bar{N}_{*}(r, a ; g, f) \quad$ and $\quad \bar{N}_{*}(r, a ; f, g)=\bar{N}_{L}(r, a ; f)+\bar{N}_{L}(r, a ; g)$.

\section{Lemmas}

Let $F$ and $G$ be two non-constant meromorphic functions defined in $\mathbf{C}$. We denote by $H$ and $V$ the functions as follows:

$$
\begin{aligned}
& H=\left(\frac{F^{\prime \prime}}{F^{\prime}}-\frac{2 F^{\prime}}{F-1}\right)-\left(\frac{G^{\prime \prime}}{G^{\prime}}-\frac{2 G^{\prime}}{G-1}\right) . \\
& V=\left(\frac{F^{\prime}}{F-1}-\frac{F^{\prime}}{F}\right)-\left(\frac{G^{\prime}}{G-1}-\frac{G^{\prime}}{G}\right) .
\end{aligned}
$$

Lemma 1 [18]. Let $f$ be a non-constant meromorphic function and let $a_{n}(z)(\not \equiv 0), a_{n-1}(z), \ldots, a_{0}(z)$ be meromorphic functions such that $T\left(r, a_{i}(z)\right)=$ $S(r, f)$ for $i=0,1,2, \ldots, n$. Then

$$
T\left(r, a_{n} f^{n}+a_{n-1} f^{n-1}+\cdots+a_{1} f+a_{0}\right)=n T(r, f)+S(r, f) .
$$

Lemma 2 [23]. Let $f$ be a non-constant meromorphic function and $p, k$ be positive integers. Then

$$
\begin{gathered}
N_{p}\left(r, 0 ; f^{(k)}\right) \leq T\left(r, f^{(k)}\right)-T(r, f)+N_{p+k}(r, 0 ; f)+S(r, f), \\
N_{p}\left(r, 0 ; f^{(k)}\right) \leq k \bar{N}(r, \infty ; f)+N_{p+k}(r, 0 ; f)+S(r, f) .
\end{gathered}
$$

Lemma 3 [12]. If $N\left(r, 0 ; f^{(k)} \mid f \neq 0\right)$ denotes the counting function of those zeros of $f^{(k)}$ which are not the zeros of $f$, where a zero of $f^{(k)}$ is counted according to its multiplicity, then

$$
N\left(r, 0 ; f^{(k)} \mid f \neq 0\right) \leq k \bar{N}(r, \infty ; f)+N(r, 0 ; f \mid<k)+k \bar{N}(r, 0 ; f \mid \geq k)+S(r, f) .
$$

Lemma 4 [[7], Theorem 3.10]. Suppose that $f$ is a non-constant meromorphic function, $k \geq 2$ is an integer. If

$$
N(r, \infty, f)+N(r, 0 ; f)+N\left(r, 0 ; f^{(k)}\right)=S\left(r, \frac{f^{\prime}}{f}\right),
$$

then $f=e^{a z+b}$, where $a \neq 0, b$ are constants. 
Lemma 5 [6]. Let $f(z)$ be a non-constant entire function and let $k \geq 2$ be a positive integer. If $f(z) f^{(k)}(z) \neq 0$, then $f(z)=e^{a z+b}$, where $a \neq 0, b$ are constant.

Lemma 6 [[22], Theorem 1.24]. Let $f$ be a non-constant meromorphic function and let $k$ be a positive integer. Suppose that $f^{(k)} \not \equiv 0$, then

$$
N\left(r, 0 ; f^{(k)}\right) \leq N(r, 0 ; f)+k \bar{N}(r, \infty ; f)+S(r, f) .
$$

LEMMA 7. Let $f$ and $g$ be two non-constant meromorphic functions and $P(\omega)=a_{m} \omega^{m}+a_{m-1} \omega^{m-1}+\cdots+a_{1} \omega+a_{0}$ or $P(\omega)=C$, where $a_{0}, a_{1}, \ldots, a_{m-1}$, $a_{m}(\neq 0), C(\neq 0)$ are complex constants. Let $n(\geq 1), k(\geq 1)$ and $m^{* *}(\geq 0)$ be three integers such that $n>3 k+m^{* *}+1$. If $\left[f^{n} P(f)\right]^{(k)} \equiv\left[g^{n} P(g)\right]^{(k)}$, then $f^{n} P(f) \equiv$ $g^{n} P(g)$.

Proof. We have $\left[f^{n} P(f)\right]^{(k)} \equiv\left[g^{n} P(g)\right]^{(k)}$.

Integrating we get

$$
\left[f^{n} P(f)\right]^{(k-1)} \equiv\left[g^{n} P(g)\right]^{(k-1)}+c_{k-1} .
$$

If possible suppose $c_{k-1} \neq 0$. Now in the view of Lemma 2 for $p=1$ and using the second fundamental theorem we get

$$
\begin{aligned}
&\left(n+m^{* *}\right) T(r, f) \\
& \leq T\left(r,\left[f^{n} P(f)\right]^{(k-1)}\right)-\bar{N}\left(r, 0 ;\left[f^{n} P(f)\right]^{(k-1)}\right)+N_{k}\left(r, 0 ; f^{n} P(f)\right)+S(r, f) \\
& \leq \bar{N}\left(r, 0 ;\left[f^{n} P(f)\right]^{(k-1)}\right)+\bar{N}(r, \infty ; f)+\bar{N}\left(r, c_{k-1} ;\left[f^{n} P(f)\right]^{(k-1)}\right) \\
&-\bar{N}\left(r, 0 ;\left[f^{n} P(f)\right]^{(k-1)}\right)+N_{k}\left(r, 0 ; f^{n} P(f)\right)+S(r, f) \\
& \leq \bar{N}(r, \infty ; f)+\bar{N}\left(r, 0 ;\left[g^{n} P(g)\right]^{(k-1)}\right)+k \bar{N}(r, 0 ; f)+N(r, 0 ; P(f))+S(r, f) \\
& \leq\left(k+1+m^{* *}\right) T(r, f)+(k-1) \bar{N}(r, \infty ; g)+N_{k}\left(r, 0 ; g^{n} P(g)\right)+S(r, f) \\
& \leq\left(k+1+m^{* *}\right) T(r, f)+k \bar{N}(r, \infty ; g)+k \bar{N}(r, 0 ; g)+N(r, 0 ; P(g))+S(r, f) \\
& \leq\left(k+1+m^{* *}\right) T(r, f)+\left(2 k+m^{* *}\right) T(r, g)+S(r, f)+S(r, g) \\
& \leq\left(3 k+2 m^{* *}+1\right) T(r)+S(r) .
\end{aligned}
$$

Similarly we get

$$
\left(n+m^{* *}\right) T(r, g) \leq\left(3 k+2 m^{* *}+1\right) T(r)+S(r) .
$$


Combining these we get

$$
\left(n-m^{* *}-3 k-1\right) T(r) \leq S(r),
$$

which is a contradiction since $n>3 k+m^{* *}+1$.

Therefore $c_{k-1}=0$ and so $\left[f^{n} P(f)\right]^{(k-1)} \equiv\left[g^{n} P(g)\right]^{(k-1)}$.

Proceeding in this way we obtain

$$
\left[f^{n} P(f)\right]^{\prime} \equiv\left[g^{n} P(g)\right]^{\prime}
$$

Integrating we get

$$
f^{n} P(F) \equiv g^{n} P(g)+c_{0} .
$$

If possible suppose $c_{0} \neq 0$. Now using the second fundamental theorem we get

$$
\begin{aligned}
\left(n+m^{* *}\right) T(r, f) \leq & \bar{N}\left(r, 0 ; f^{n} P(f)\right)+\bar{N}\left(r, \infty ; f^{n} P(f)\right)+\bar{N}\left(r, c_{0} ; f^{n} P(f)\right) \\
\leq & \bar{N}(r, 0 ; f)+m^{* *} T(r, f)+\bar{N}(r, \infty ; f)+\bar{N}\left(r, 0 ; g^{n} P(g)\right) \\
\leq & \left(m^{* *}+1\right) T(r, f)+\bar{N}(r, \infty ; f) \\
& +\bar{N}(r, 0 ; g)+m^{* *} T(r, g)+S(r, f) \\
\leq & \left(3+2 m^{* *}\right) T(r)+S(r) .
\end{aligned}
$$

Similarly we get

$$
\left(n+m^{* *}\right) T(r, g) \leq\left(3+2 m^{* *}\right) T(r)+S(r) .
$$

Combining these we get

$$
\left(n-3-m^{* *}\right) T(r) \leq S(r),
$$

which is a contradiction since $n>4+m^{* *}$.

Therefore $c_{0}=0$ and so

$$
f^{n} P(f) \equiv g^{n} P(g)
$$

This proves the Lemma.

Lemma 8. Let $f, g$ be two non-constant meromorphic functions and $P(\omega)=$ $a_{m} \omega^{m}+a_{m-1} \omega^{m-1}+\cdots+a_{1} \omega+a_{0}$ or $P(\omega)=C$, where $a_{0}, a_{1}, \ldots, a_{m-1}, a_{m}(\neq 0)$, $C(\neq 0)$ are complex constants. Let $n(\geq 1), m^{* *}(\geq 0)$ and $k(\geq 1)$ be three integers with $n>k+2$. If $\left[f^{n} P(f)\right]^{(k)}$ and $\left[g^{n} P(g)\right]^{(k)}$ share $\alpha$ IM, where $\alpha(\not \equiv 0, \infty)$ is a small function of $f$ and $g$, then $T(r, f)=O(T(r, g))$ and $T(r, g)=O(T(r, f))$. 
Proof. Let $F=f^{n} P(f)$. By the second fundamental theorem for small functions $\{$ see $[20]\}$, we have

$$
T\left(r, F^{(k)}\right) \leq \bar{N}\left(r, \infty ; F^{(k)}\right)+\bar{N}\left(r, 0 ; F^{(k)}\right)+\bar{N}\left(r, \alpha ; F^{(k)}\right)+(\varepsilon+o(1)) T(r, F),
$$

for all $\varepsilon>0$.

Now in the view of Lemmas 1 and 2 for $p=1$ and using above we get

$$
\begin{aligned}
\left(n+m^{* *}\right) T(r, f) \leq & T\left(r,\left[f^{n} P(f)\right]^{(k)}\right)-\bar{N}\left(r, 0 ;\left[f^{n} P(f)\right]^{(k)}\right) \\
& +N_{k+1}\left(r, 0 ; f^{n} P(f)\right)+(\varepsilon+o(1)) T(r, f) \\
\leq & \bar{N}\left(r, 0 ;\left[f^{n} P(f)\right]^{(k)}\right)+\bar{N}(r, \infty ; f)+\bar{N}\left(r, \alpha ;\left[f^{n} P(f)\right]^{(k)}\right) \\
& -\bar{N}\left(r, 0 ;\left[f^{n} P(f)\right]^{(k)}\right)+N_{k+1}\left(r, 0 ; f^{n} P(f)\right)+(\varepsilon+o(1)) T(r, f) \\
\leq & \bar{N}(r, \infty ; f)+\bar{N}\left(r, \alpha ;\left[f^{n} P(f)\right]^{(k)}\right)+(k+1) \bar{N}(r, 0 ; f) \\
& +N(r, 0 ; P(f))+(\varepsilon+o(1)) T(r, f) \\
\leq & \left(k+2+m^{* *}\right) T(r, f)+\bar{N}\left(r, \alpha ;\left[g^{n} P(g)\right]^{(k)}\right)+(\varepsilon+o(1)) T(r, f) \\
\leq & \left(k+2+m^{* *}\right) T(r, f)+(k+1)\left(n+m^{* *}\right) T(r, g) \\
& +(\varepsilon+o(1)) T(r, f),
\end{aligned}
$$

i.e.,

$$
(n-k-2) T(r, f) \leq(k+1)\left(n+m^{* *}\right) T(r, g)+(\varepsilon+o(1)) T(r, f) .
$$

Since $n>k+2$, take $\varepsilon<1$ and we have $T(r, f)=O(T(r, g))$. Similarly we have $T(r, g)=O(T(r, f))$. This completes the proof of the Lemma.

Lemma 9. Let $f, g$ be two transcendental meromorphic functions and $P(\omega)=$ $a_{m} \omega^{m}+a_{m-1} \omega^{m-1}+\cdots+a_{1} \omega+a_{0}$ or $P(\omega)=C$, where $a_{0}, a_{1}, \ldots, a_{m-1}, a_{m}(\neq 0)$, $C(\neq 0)$ are complex constants. Let $F=\frac{\left[f^{n} P(f)\right]^{(k)}}{p}, G=\frac{\left[g^{n} P(g)\right]^{(k)}}{p}$, where $p(z)$ is a non zero polynomial and $n(\geq 1), k(\geq 1)$ and $m^{* *}(\geq 0)$ are integers such that $n>3 k+m^{* *}+3$. If $f, g$ share $\infty I M$ and $H \equiv 0$, then one of the following two cases holds:

(i) $\left[f^{n} P(f)\right]^{(k)}\left[g^{n} P(f)\right]^{(k)} \equiv p^{2}$, where $\left[f^{n} P(f)\right]^{(k)}-p(z)$ and $\left[g^{n} P(g)\right]^{(k)}-$ $p(z)$ share 0 CM;

(ii) $f^{n} P(f) \equiv g^{n} P(g)$. 
Proof. Since $H \equiv 0$, on integration we get

$$
\frac{1}{F-1} \equiv \frac{b G+a-b}{G-1}
$$

where $a, b$ are constants and $a \neq 0$. From (2.5) it follows that $F$ and $G$ share $1 \mathrm{CM}$. We now consider the following cases:

Case 1. Let $b \neq 0$ and $a \neq b$.

If $b=-1$, then from (2.5) we have

$$
F \equiv \frac{-a}{G-a-1}
$$

Therefore

$$
\bar{N}(r, a+1 ; G)=\bar{N}(r, \infty ; F)=\bar{N}(r, \infty ; f) .
$$

So in view of Lemmas 1 and 2 for $p=1$ and using the second fundamental theorem we get

$$
\begin{aligned}
\left(n+m^{* *}\right) T(r, g) \leq & T(r, G)+N_{k+1}\left(r, 0 ; g^{n} P(g)\right)-\bar{N}(r, 0 ; G) \\
\leq & \bar{N}(r, \infty ; G)+\bar{N}(r, 0 ; G)+\bar{N}(r, a+1 ; G) \\
& +N_{k+1}\left(r, 0 ; g^{n} P(g)\right)-\bar{N}(r, 0 ; G)+S(r, g) \\
\leq & \bar{N}(r, \infty ; g)+N_{k+1}\left(r, 0 ; g^{n} P(g)\right)+\bar{N}(r, \infty ; f)+S(r, g) \\
\leq & \bar{N}(r, \infty ; f)+\bar{N}(r, \infty ; g)+N_{k+1}\left(r, 0 ; g^{n}\right) \\
& +N_{k+1}(r, 0 ; P(g))+S(r, g) \\
\leq & 2 \bar{N}(r, \infty ; g)+(k+1) \bar{N}(r, 0 ; g)+T(r, P(g))+S(r, g) \\
\leq & \left\{k+3+m^{* *}\right\} T(r, g)+S(r, g),
\end{aligned}
$$

which is a contradiction since $n>k+3$.

If $b \neq-1$, from (2.5) we obtain that

$$
F-\left(1+\frac{1}{b}\right) \equiv \frac{-a}{b^{2}\left[G+\frac{a-b}{b}\right]} .
$$

So

$$
\bar{N}\left(r, \frac{(b-a)}{b} ; G\right)=\bar{N}(r, \infty ; F)=\bar{N}(r, \infty ; f) .
$$


Using Lemmas 1, 2 and the same argument as used in the case when $b=-1$ we can get a contradiction.

Case 2. Let $b \neq 0$ and $a=b$.

If $b=-1$, then from (2.5) we have

$$
F G \equiv 1
$$

i.e.,

$$
\left[f^{n} P(f)\right]^{(k)}\left[g^{n} P(g)\right]^{(k)} \equiv p^{2},
$$

where $\left[f^{n} P(f)\right]^{(k)}-p(z)$ and $\left[g^{n} P(g)\right]^{(k)}-p(z)$ share $0 \mathrm{CM}$.

If $b \neq-1$, from $(2.5)$ we have

$$
\frac{1}{F} \equiv \frac{b G}{(1+b) G-1} .
$$

Therefore

$$
\bar{N}\left(r, \frac{1}{1+b} ; G\right)=\bar{N}(r, 0 ; F) .
$$

So in view of Lemmas 1 and 2 for $p=1$ and using the second fundamental theorem we get

$$
\begin{aligned}
\left(n+m^{* *}\right) T(r, g) \leq & \bar{N}(r, \infty ; G)+\bar{N}(r, 0 ; G)+\bar{N}\left(r, \frac{1}{1+b} ; G\right) \\
& +N_{k+1}\left(r, 0 ; g^{n} P(g)\right)-\bar{N}(r, 0 ; G)+S(r, g) \\
\leq & \bar{N}(r, \infty ; g)+(k+1) \bar{N}(r, 0 ; g)+T(r, P(g))+\bar{N}(r, 0 ; F)+S(r, g) \\
\leq & \bar{N}(r, \infty ; g)+(k+1) \bar{N}(r, 0 ; g)+T(r, P(g))+(k+1) \bar{N}(r, 0 ; f) \\
& +T(r, P(f))+k \bar{N}(r, \infty ; f)+S(r, f)+S(r, g) \\
\leq & \left\{k+2+m^{* *}\right\} T(r, g)+\left\{2 k+1+m^{* *}\right\} T(r, f) \\
& +S(r, f)+S(r, g) .
\end{aligned}
$$

Without loss of generality, we suppose that there exists a set $I$ with infinite measure such that $T(r, f) \leq T(r, g)$ for $r \in I$.

So for $r \in I$ we have

$$
\left\{n-3 k-3-m^{* *}\right\} T(r, g) \leq S(r, g),
$$

which is a contradiction since $n>3 k+3+m^{* *}$. 
Case 3. Let $b=0$. From (2.5) we obtain

$$
F \equiv \frac{G+a-1}{a} .
$$

If $a \neq 1$ then from (2.6) we obtain

$$
\bar{N}(r, 1-a ; G)=\bar{N}(r, 0 ; F) .
$$

We can similarly deduce a contradiction as in Case 2. Therefore $a=1$ and from (2.6) we obtain

$$
F \equiv G
$$

i.e.,

$$
\left[f^{n} P(f)\right]^{(k)} \equiv\left[g^{n} P(g)\right]^{(k)} .
$$

Then by Lemma 7 we have

$$
f^{n} P(f) \equiv g^{n} P(g)
$$

This completes the proof.

Lemma 10 [22]. Let $f_{j}(j=1,2,3)$ be a meromorphic and $f_{1}$ be non-constant. Suppose that

$$
\sum_{j=1}^{3} f_{j} \equiv 1
$$

and

$$
\sum_{j=1}^{3} N\left(r, 0 ; f_{j}\right)+2 \sum_{j=1}^{3} \bar{N}\left(r, \infty ; f_{j}\right)<(\lambda+o(1)) T(r),
$$

as $r \rightarrow+\infty, r \in I, \lambda<1$ and $T(r)=\max _{1 \leq j \leq 3} T\left(r, f_{j}\right)$. Then $f_{2} \equiv 1$ or $f_{3} \equiv 1$.

Lemma 11. Let $f, g$ be two transcendental meromorphic functions, $p(z)$ be a non-zero polynomial with $\operatorname{deg}(p) \leq n-1$, where $n$ and $k$ be two positive integers such that $n>\max \{2 k, k+2\}$. Suppose $\left[f^{n}\right]^{(k)}\left[g^{n}\right]^{(k)} \equiv p^{2}$, where $\left[f^{n}\right]^{(k)}-p(z)$, $\left[g^{n}\right]^{(k)}-p(z)$ share $0 C M$ and $f, g$ share $\infty I M$,

(i) if $p(z)$ is not a constant, then $f=c_{1} e^{c Q(z)}, g=c_{2} e^{-c Q(z)}$, where $Q(z)=$ $\int_{0}^{z} p(z) d z, c_{1}, c_{2}$ and $c$ are constants such that $(n c)^{2}\left(c_{1} c_{2}\right)^{n}=-1$, 
(ii) if $p(z)$ is a nonzero constant $b$, then $f=c_{3} e^{d z}, g=c_{4} e^{-d z}$, where $c_{3}, c_{4}$ and $d$ are constants such that $(-1)^{k}\left(c_{3} c_{4}\right)^{n}(n d)^{2 k}=b^{2}$.

Proof. Suppose

$$
\left[f^{n}\right]^{(k)}\left[g^{n}\right]^{(k)} \equiv p^{2}
$$

Since $f$ and $g$ share $\infty$ IM, (2.7) one can easily say that $f$ and $g$ are transcendental entire functions.

We consider the following cases:

Case 1: Let $\operatorname{deg}(p(z))=l(\geq 1)$.

By Lemma 8 we have $S(r, f)=S(r, g)$. At first we observe that $f$ and $g$ being two transcendental meromorphic functions $N(r, 0 ; f)=N(r, 0 ; g)=O(\log r)$ $=S(r, f)=S(r, g)$.

Let

$$
F_{1}=\frac{\left[f^{n}\right]^{(k)}}{p} \text { and } G_{1}=\frac{\left[g^{n}\right]^{(k)}}{p} .
$$

Note that $T\left(r, F_{1}\right) \leq n(k+1) T(r, f)+S(r, f)$ and so $T\left(r, F_{1}\right)=O(T(r, f))$. Also by Lemma 2, one can obtain $T(r, f)=O\left(T\left(r, F_{1}\right)\right)$. Hence $S\left(r, F_{1}\right)=S(r, f)$. Similarly we get $S\left(r, G_{1}\right)=S(r, g)$. Hence we get $S\left(r, F_{1}\right)=S\left(r, G_{1}\right)$. From (2.7) we get

$$
F_{1} G_{1} \equiv 1
$$

If $F_{1} \equiv c G_{1}$, where $c$ is a nonzero constant, then by (2.9), $F_{1}$ is a constant and so $f$ is a polynomial, which contradicts our assumption. Hence $F_{1} \not \equiv c G_{1}$.

Let

$$
\Phi=\frac{\left[f^{n}\right]^{(k)}-p}{\left[g^{n}\right]^{(k)}-p} .
$$

We deduce from (2.10) that

$$
\Phi \equiv e^{\beta}
$$

where $\beta$ is an entire function.

Let $f_{1}=F_{1}, f_{2}=-e^{\beta} G_{1}$ and $f_{3}=e^{\beta}$. Here $f_{1}$ is transcendental. Now from (2.11), we have

$$
f_{1}+f_{2}+f_{3} \equiv 1
$$


Hence by Lemma 6 we get

$$
\begin{aligned}
\sum_{j=1}^{3} N\left(r, 0 ; f_{j}\right)+2 \sum_{j=1}^{3} \bar{N}\left(r, \infty ; f_{j}\right) & \leq N\left(r, 0 ; F_{1}\right)+N\left(r, 0 ; e^{\beta} G_{1}\right)+O(\log r) \\
& \leq(\lambda+o(1)) T(r),
\end{aligned}
$$

as $r \rightarrow+\infty, r \in I, \lambda<1$ and $T(r)=\max _{1 \leq j \leq 3} T\left(r, f_{j}\right)$.

So by Lemma 10, we get either $e^{\beta} G_{1} \equiv-1$ or $e^{\beta} \equiv 1$. But here the only possibility is that $e^{\beta} G_{1} \equiv-1$, i.e., $\left[g^{n}\right]^{(k)} \equiv-e^{-\beta} p(z)$ and so from (2.7) we obtain

$$
F_{1} \equiv e^{\gamma_{1}} G_{1}
$$

i.e.,

$$
\left[f^{n}\right]^{(k)} \equiv e^{\gamma_{1}}\left[g^{n}\right]^{(k)},
$$

where $\gamma_{1}$ is a non-constant entire function. Now from (2.7) we get

$$
\left(f^{n}\right)^{k} \equiv c e^{(1 / 2) \gamma_{1}} p(z), \quad\left(g^{n}\right)^{k} \equiv c e^{-(1 / 2) \gamma_{1}} p(z),
$$

where $c= \pm 1$.

Since $N(r, 0 ; f)=O(\log r)$ and $N(r, 0 ; g)=O(\log r)$, so we can take

$$
f(z)=h_{1}(z) e^{\alpha(z)}, \quad g(z)=h_{2}(z) e^{\beta(z)},
$$

where $h_{1}$ and $h_{2}$ are nonzero polynomials and $\alpha, \beta$ are two non-constant entire functions.

We deduce from (2.7) and (2.13) that either both $\alpha$ and $\beta$ are transcendental entire functions or both are polynomials.

We consider the following cases:

Subcase 1.1: Let $k \geq 2$.

First we suppose both $\alpha$ and $\beta$ are transcendental entire functions.

Let $\alpha_{1}=\alpha^{\prime}+\frac{h_{1}^{\prime}}{h_{1}}$ and $\beta_{1}=\beta^{\prime}+\frac{h_{2}^{\prime}}{h_{2}}$. Clearly both $\alpha_{1}$ and $\beta_{1}$ are transcendental functions.

Note that

$$
S\left(r, n \alpha_{1}\right)=S\left(r, \frac{\left[f^{n}\right]^{\prime}}{f^{n}}\right), \quad S\left(r, n \beta_{1}\right)=S\left(r, \frac{\left[g^{n}\right]^{\prime}}{g^{n}}\right) .
$$

Moreover we see that

$$
\begin{gathered}
N\left(r, 0 ;\left[f^{n}\right]^{(k)}\right) \leq N\left(r, 0 ; p^{2}\right)=O(\log r) . \\
N\left(r, 0 ;\left[g^{n}\right]^{(k)}\right) \leq N\left(r, 0 ; p^{2}\right)=O(\log r) .
\end{gathered}
$$


From these and using (2.13) we have

$$
N\left(r, \infty ; f^{n}\right)+N\left(r, 0 ; f^{n}\right)+N\left(r, 0 ;\left[f^{n}\right]^{(k)}\right)=S\left(r, n \alpha_{1}\right)=S\left(r, \frac{\left[f^{n}\right]^{\prime}}{f^{n}}\right)
$$

and

$$
N\left(r, \infty ; g^{n}\right)+N\left(r, 0 ; g^{n}\right)+N\left(r, 0 ;\left[g^{n}\right]^{(k)}\right)=S\left(r, n \beta_{1}\right)=S\left(r, \frac{\left[g^{n}\right]^{\prime}}{g^{n}}\right) .
$$

Then from (2.14), (2.15) and Lemma 4 we must have

$$
f=e^{a z+b}, \quad g=e^{c z+d},
$$

where $a \neq 0, b, c \neq 0$ and $d$ are constants. But these types of $f$ and $g$ do not agree with the relation (2.7).

Next we suppose $\alpha$ and $\beta$ are both non-constant polynomials, since otherwise $f, g$ reduces to a polynomials contradicting that they are transcendental.

Also from (2.7) we get $\alpha+\beta \equiv C$ i.e., $\alpha^{\prime} \equiv-\beta^{\prime}$. Therefore $\operatorname{deg}(\alpha)=\operatorname{deg}(\beta)$.

Suppose $h_{i}$ 's $i=1,2$ are non-constant polynomials. We deduce from (2.13) that

$$
\left[f^{n}\right]^{(k)} \equiv A h_{1}^{n-k}\left[h_{1}^{k}\left(\alpha^{\prime}\right)^{k}+P_{k-1}\left(\alpha^{\prime}, h_{1}^{\prime}\right)\right] e^{n \alpha} \equiv p(z) e^{n \alpha},
$$

and

$$
\left[g^{n}\right]^{(k)} \equiv B h_{2}^{n-k}\left[h_{2}^{k}\left(\beta^{\prime}\right)^{k}+Q_{k-1}\left(\beta^{\prime}, h_{2}^{\prime}\right)\right] e^{n \beta} \equiv p(z) e^{n \beta},
$$

where $A, B$ are nonzero constants, $P_{k-1}\left(\alpha^{\prime}, h_{1}^{\prime}\right)$ and $Q_{k-1}\left(\beta^{\prime}, h_{2}^{\prime}\right)$ are differential polynomials in $\alpha^{\prime}, h_{1}^{\prime}$ and $\beta^{\prime}, h_{2}^{\prime}$ respectively.

Since $\operatorname{deg}(p) \leq n-1$, from (2.17) and (2.18) we conclude that both $h_{1}$ and $h_{2}$ are nonzero constants.

So we can rewrite $f$ and $g$ as follows:

$$
f=e^{\gamma}, \quad g=e^{\delta} .
$$

We deduce from (2.19) that

$$
\begin{gathered}
\left(f^{n}\right)^{\prime}=n \gamma^{\prime} e^{n \gamma} \\
\left(f^{n}\right)^{\prime \prime}=\left[n^{2}\left(\gamma^{\prime}\right)^{2}+n \gamma^{\prime \prime}\right] e^{n \gamma} \\
\left(f^{n}\right)^{\prime \prime \prime}=\left[n^{3}\left(\gamma^{\prime}\right)^{3}+3 n^{2} \gamma^{\prime} \gamma^{\prime \prime}+n \gamma^{\prime \prime \prime}\right] e^{n \gamma} \\
\left(f^{n}\right)^{(i v)}=\left[n^{4}\left(\gamma^{\prime}\right)^{4}+6 n^{3}\left(\gamma^{\prime}\right)^{2} \gamma^{\prime \prime}+3 n^{2}\left(\gamma^{\prime \prime}\right)^{2}+4 n^{2} \gamma^{\prime} \gamma^{\prime \prime \prime}+n \gamma^{(i v)}\right] e^{n \gamma}
\end{gathered}
$$




$$
\begin{aligned}
& \left(f^{n}\right)^{(v)}=\left[n^{5}\left(\gamma^{\prime}\right)^{5}+10 n^{4}\left(\gamma^{\prime}\right)^{3} \gamma^{\prime \prime}+15 n^{3} \gamma^{\prime}\left(\gamma^{\prime \prime}\right)^{2}+10 n^{3}\left(\gamma^{\prime}\right)^{2} \gamma^{\prime \prime \prime}\right. \\
& \left.+10 n^{2} \gamma^{\prime \prime} \gamma^{\prime \prime \prime}+5 n^{2} \gamma^{\prime} \gamma^{(i v)}+n \gamma^{(v)}\right] e^{n \gamma} \\
& {\left[f^{n}\right]^{(k)}=\left[n^{k}\left(\gamma^{\prime}\right)^{k}+K\left(\gamma^{\prime}\right)^{k-2} \gamma^{\prime \prime}+P_{k-2}\left(\gamma^{\prime}\right)\right] e^{n \gamma} .}
\end{aligned}
$$

Similarly we get

$$
\begin{aligned}
{\left[g^{n}\right]^{(k)} } & =\left[n^{k}\left(\delta^{\prime}\right)^{k}+K\left(\delta^{\prime}\right)^{k-2} \delta^{\prime \prime}+P_{k-2}\left(\delta^{\prime}\right)\right] e^{n \delta} \\
& =\left[(-1)^{k} n^{k}\left(\gamma^{\prime}\right)^{k}-K(-1)^{k-2}\left(\gamma^{\prime}\right)^{k-2} \gamma^{\prime \prime}+P_{k-2}\left(-\gamma^{\prime}\right)\right] e^{n \delta},
\end{aligned}
$$

where $K$ is a suitably positive integer and $P_{k-2}\left(\gamma^{\prime}\right)$ is a differential polynomial in $\gamma^{\prime}$.

Since $\operatorname{deg}(\gamma) \geq 2$, we observe that $\operatorname{deg}\left(\left(\gamma^{\prime}\right)^{k}\right) \geq k \operatorname{deg}\left(\gamma^{\prime}\right)$ and so $\left(\gamma^{\prime}\right)^{k-2} \gamma^{\prime \prime}$ is either a nonzero constant or $\operatorname{deg}\left(\left(\gamma^{\prime}\right)^{k-2} \gamma^{\prime \prime}\right) \geq(k-1) \operatorname{deg}\left(\gamma^{\prime}\right)-1$. Also we see that

$$
\operatorname{deg}\left(\left(\gamma^{\prime}\right)^{k}\right)>\operatorname{deg}\left(\left(\gamma^{\prime}\right)^{k-2} \gamma^{\prime \prime}\right)>\operatorname{deg}\left(P_{k-2}\left(\gamma^{\prime}\right)\right)\left(\text { or } \operatorname{deg}\left(P_{k-2}\left(-\gamma^{\prime}\right)\right)\right) .
$$

Now from (2.12) we see that $\left[f^{n}\right]^{(k)}$ and $\left[g^{n}\right]^{(k)}$ share $0 \mathrm{CM}$ and so the polynomials

$$
n^{k}\left(\gamma^{\prime}\right)^{k}+K\left(\gamma^{\prime}\right)^{k-2} \gamma^{\prime \prime}+P_{k-2}\left(\gamma^{\prime}\right)
$$

and

$$
(-1)^{k} n^{k}\left(\gamma^{\prime}\right)^{k}-K(-1)^{k-2}\left(\gamma^{\prime}\right)^{k-2} \gamma^{\prime \prime}+P_{k-2}\left(-\gamma^{\prime}\right)
$$

must be identical but this is impossible for $k \geq 2$.

Actually the terms $n^{k}\left(\gamma^{\prime}\right)^{k}+K\left(\gamma^{\prime}\right)^{k-2} \gamma^{\prime \prime}$ and $(-1)^{k} n^{k}\left(\gamma^{\prime}\right)^{k}-K(-1)^{k-2}$. $\left(\gamma^{\prime}\right)^{k-2} \gamma^{\prime \prime}$ can not be identical for $k \geq 2$.

Subcase 1.2: Let $k=1$.

Now from (2.7) we get

$$
f^{n-1} f^{\prime} g^{n-1} g^{\prime} \equiv p_{1}^{2}
$$

where $p_{1}^{2}=\frac{1}{n^{2}} p^{2}$.

We wish to prove that both $\alpha$ and $\beta$ are polynomials.

To this end let $h=f g$ and suppose at least one of $\alpha$ and $\beta$ is a transcendental entire function. We consider the following subcases:

Subcase 1.2.1: First suppose that $h$ is a polynomial. Then from (2.13), it is clear that $h=A h_{1} h_{2}$, where $A=e^{C}$ and $\alpha+\beta \equiv C$ a constant. It follwos that both $\alpha$ and $\beta$ are transcendental. Therefore $\alpha^{\prime} \equiv-\beta^{\prime}$. 
Now from (2.20) we see that

$$
A \alpha^{\prime}\left(-h_{1}^{\prime} h_{2}+h_{1} h_{2}^{\prime}-h_{1} h_{2} \alpha^{\prime}\right) \equiv e^{-(n-1) C} \frac{p_{1}^{2}}{\left(h_{1} h_{2}\right)^{n-1}}-A h_{1}^{\prime} h_{2}^{\prime},
$$

where $\frac{p_{1}^{2}}{\left(h_{1} h_{2}\right)^{n-1}}$ is a polynomial. From this it is clear that

$$
N\left(r, 0 ; \alpha^{\prime}\right)=O(\log r), \quad N\left(r, 0 ;-h_{1}^{\prime} h_{2}+h_{1} h_{2}^{\prime}-h_{1} h_{2} \alpha^{\prime}\right)=O(\log r) .
$$

By the second fundamental theorem for small functions $\{$ see $[20]\}$, we have

$$
\begin{aligned}
T\left(r, \alpha^{\prime}\right) \leq & \bar{N}\left(r, \infty ; \alpha^{\prime}\right)+\bar{N}\left(r, 0 ; \alpha^{\prime}\right)+\bar{N}\left(r, 0 ;-h_{1}^{\prime} h_{2}+h_{1} h_{2}^{\prime}-h_{1} h_{2} \alpha^{\prime}\right) \\
& +(\varepsilon+o(1)) T\left(r, \alpha^{\prime}\right) \\
\leq & O(\log r)+(\varepsilon+o(1)) T\left(r, \alpha^{\prime}\right)
\end{aligned}
$$

for all $\varepsilon>0$. This shows that $\alpha^{\prime}$ is a polynomial and so is $\alpha$, which is a contradiction.

Subcase 1.2.2: Next suppose $h$ is a transcendental entire function. From (2.20) we get

$$
\left(\frac{g^{\prime}}{g}-\frac{1}{2} \frac{h^{\prime}}{h}\right)^{2} \equiv \frac{1}{4}\left(\frac{h^{\prime}}{h}\right)^{2}-h^{-n} p_{1}^{2}
$$

Let

$$
\alpha_{2}=\frac{g^{\prime}}{g}-\frac{1}{2} \frac{h^{\prime}}{h}
$$

From (2.21) we get

$$
\alpha_{2}^{2} \equiv \frac{1}{4}\left(\frac{h^{\prime}}{h}\right)^{2}-h^{-n} p_{1}^{2}
$$

First we suppose $\alpha_{2} \equiv 0$. Then we get $h^{-n} p_{1}^{2} \equiv \frac{1}{4}\left(\frac{h^{\prime}}{h}\right)^{2}$ and so $T(r, h)=S(r, h)$, which is impossible. Next we suppose that $\alpha_{2} \not \equiv 0$. Differentiating (2.22) we get

$$
2 \alpha_{2} \alpha_{2}^{\prime} \equiv \frac{1}{2} \frac{h^{\prime}}{h}\left(\frac{h^{\prime}}{h}\right)^{\prime}+n h^{\prime} h^{-n-1} p_{1}^{2}-2 h^{-n} p_{1} p_{1}^{\prime} .
$$

Applying (2.22) we obtain

$$
h^{-n}\left(-n \frac{h^{\prime}}{h} p_{1}^{2}+2 p_{1} p_{1}^{\prime}-2 \frac{\alpha_{2}^{\prime}}{\alpha_{2}} p_{1}^{2}\right) \equiv \frac{1}{2} \frac{h^{\prime}}{h}\left(\left(\frac{h^{\prime}}{h}\right)^{\prime}-\frac{h^{\prime}}{h} \frac{\alpha_{2}^{\prime}}{\alpha_{2}}\right) .
$$


If we suppose

$$
-n \frac{h^{\prime}}{h} p_{1}^{2}+2 p_{1} p_{1}^{\prime}-2 \frac{\alpha_{2}^{\prime}}{\alpha_{2}} p_{1}^{2} \equiv 0
$$

then there exist a non-zero constant $c$ such that $\alpha_{2}^{2} \equiv c h^{-n} p_{1}^{2}$ and so from (2.22) we get

$$
(c+1) h^{-n} p_{1}^{2} \equiv \frac{1}{4}\left(\frac{h^{\prime}}{h}\right)^{2} .
$$

If $c=-1$, then $h$ will be a constant. If $c \neq-1$, then we have $T(r, h)=S(r, h)$, which is impossible. Next we suppose that

$$
-n \frac{h^{\prime}}{h} p_{1}^{2}+2 p_{1} p_{1}^{\prime}-2 \frac{\alpha_{2}^{\prime}}{\alpha_{2}} p_{1}^{2} \not \equiv 0 .
$$

Then by (2.23) we have

$$
\begin{aligned}
n T(r, h) & =n m(r, h) \\
& \leq m\left(r, h^{n} \frac{1}{2} \frac{h^{\prime}}{h}\left(\left(\frac{h^{\prime}}{h}\right)^{\prime}-\frac{h^{\prime}}{h} \frac{\alpha_{2}^{\prime}}{\alpha_{2}}\right)\right)+m\left(r, \frac{1}{\frac{1}{2} \frac{h^{\prime}}{h}\left(\left(\frac{h^{\prime}}{h}\right)^{\prime}-\frac{h^{\prime}}{h} \frac{\alpha_{2}^{\prime}}{\alpha_{2}}\right)}\right)+O(1) \\
& \leq T\left(r, \frac{1}{2} \frac{h^{\prime}}{h}\left(\left(\frac{h^{\prime}}{h}\right)^{\prime}-\frac{h^{\prime}}{h} \frac{\alpha_{2}^{\prime}}{\alpha_{2}}\right)\right)+m\left(r, n \frac{h^{\prime}}{h} p_{1}^{2}+2 p_{1} p_{1}^{\prime}-2 \frac{\alpha_{2}^{\prime}}{\alpha_{2}} p_{1}^{2}\right) \\
& \leq \bar{N}\left(r, 0 ; \alpha_{2}\right)+S(r, h)+S\left(r, \alpha_{2}\right) .
\end{aligned}
$$

From (2.22) we get

$$
T\left(r, \alpha_{2}\right) \leq \frac{1}{2} n T(r, h)+S(r, h) .
$$

Now from (2.24) we get

$$
\frac{1}{2} n T(r, h) \leq S(r, h),
$$

which is impossible. 
So from the above two subcases we must conclude that both $\alpha$ and $\beta$ are polynomials. Also from (2.7) we can conclude that $\alpha(z)+\beta(z) \equiv C$ for a constant $C$ and so $\alpha^{\prime}(z)+\beta^{\prime}(z) \equiv 0$. We deduce from (2.7) that

$$
\left[f^{n}\right]^{\prime} \equiv n\left[h_{1}^{n} \alpha^{\prime}+h_{1}^{n-1} h_{1}^{\prime}\right] e^{n \alpha} \equiv p(z) e^{n \alpha}
$$

and

$$
\left[g^{n}\right]^{\prime}=n\left[h_{2}^{n} \beta^{\prime}+h_{2}^{n-1} h_{2}^{\prime}\right] e^{n \beta} \equiv p(z) e^{n \beta} .
$$

Since $\operatorname{deg}(p) \leq n-1$, from (2.25) and (2.26) we conclude that both $h_{1}$ and $h_{2}$ are nonzero constant.

So we can rewrite $f$ and $g$ as follows:

$$
f=e^{\gamma_{2}}, \quad g=e^{\delta_{2}} .
$$

Now from (2.7) we get

$$
n^{2} \gamma_{2}^{\prime} \delta_{2}^{\prime} e^{n(\gamma+\delta)} \equiv p^{2}
$$

Also from (2.28) we can conclude that $\gamma_{2}(z)+\delta_{2}(z) \equiv C$ for a constant $C$ and so $\gamma_{2}^{\prime}(z)+\delta_{2}^{\prime}(z) \equiv 0$. Thus from (2.28) we get $n^{2} e^{n C} \gamma_{2}^{\prime} \delta_{2}^{\prime} \equiv p^{2}(z)$. By computation we get

$$
\gamma_{2}^{\prime}=c p(z), \quad \delta_{2}^{\prime}=-c p(z)
$$

Hence

$$
\gamma_{2}=c Q(z)+b_{1}, \quad \delta_{2}=-c Q(z)+b_{2},
$$

where $Q(z)=\int_{0}^{z} p(z) d z$ and $b_{1}, b_{2}$ are constants. Finally we take $f$ and $g$ as

$$
f(z)=c_{1} e^{c Q(z)}, \quad g(z)=c_{2} e^{-c Q(z)},
$$

where $c_{1}, c_{2}$ and $c$ are constants such that $(n c)^{2}\left(c_{1} c_{2}\right)^{n}=-1$.

Case 2: Let $p(z)$ be a nonzero constant $b$.

In this case we see that $f$ and $g$ have no zeros and so we can take $f$ and $g$ as follows:

$$
f=e^{\alpha}, \quad g=e^{\beta},
$$

where $\alpha(z), \beta(z)$ are two non-constant entire functions.

We now consider the following two subcases:

Subcase 2.1: Let $k \geq 2$.

We see that

$$
N\left(r, 0 ;\left[f^{n}\right]^{(k)}\right)=0 .
$$


From this and using (2.31) we have

$$
f^{n}(z)\left[f^{n}(z)\right]^{(k)} \neq 0 .
$$

Similarly we have

$$
g^{n}(z)\left[g^{n}(z)\right]^{(k)} \neq 0
$$

Then from (2.32), (2.33) and Lemma 5 we must have

$$
f=e^{a z+b}, \quad g=e^{c z+d},
$$

where $a \neq 0, b, c \neq 0$ and $d$ are constants. From (2.7) it is clear that $a+c=0$.

Subcase 2.1: Let $k=1$.

Considering Subcase 1.2 one can easily get

$$
f=e^{a z+b}, \quad g=e^{c z+d},
$$

where $a \neq 0, b, c \neq 0$ and $d$ are constants.

Finally we can take $f$ and $g$ as

$$
f=c_{3} e^{d z}, \quad g=c_{4} e^{-d z}
$$

where $c_{3}, c_{4}$ and $d$ are nonzero constants such that $(-1)^{k}\left(c_{3} c_{4}\right)^{n}(n d)^{2 k}=b^{2}$.

This completes the proof.

LEMMA 12. Let $f, g$ be two transcendental meromorphic functions and $P(\omega)=$ $a_{m} \omega^{m}+a_{m-1} \omega^{m-1}+\cdots+a_{1} \omega+a_{0}$ be not a monomial, where $a_{0}, a_{1}, \ldots, a_{m-1}$, $a_{m}(\neq 0)$ are complex constants. Let $n, m$ and $k$ be three positive integers such that $n>k$. If $f$ and $g$ share $\infty$ IM, then $\left[f^{n} P(f)\right]^{(k)}\left[g^{n} P(g)\right]^{(k)} \not \equiv p^{2}$, where $p(z)$ is a non zero polynomial.

Proof. Suppose

$$
\left[f^{n} P(f)\right]^{(k)}\left[g^{n} P(g)\right]^{(k)} \equiv p^{2} .
$$

For the sake of the simplicity we suppose $a_{0} \neq 0$. Since $f$ and $g$ share $\infty$ IM, (2.7) one can easily say that $f$ and $g$ are transcendental entire functions.

Since $n>k$, so we can take $f(z)$ as

$$
f(z)=h(z) e^{\alpha(z)},
$$

where $h$ is a nonzero polynomial and $\alpha$ is a non-constant entire function. 
Since $f=h e^{\alpha}$, then by induction we get

$$
a_{i}\left(f^{n+i}\right)^{(k)}=t_{i}\left(\alpha^{\prime}, \alpha^{\prime \prime}, \ldots, \alpha^{(k)}, h, h^{\prime}, \ldots, h^{(k)}\right) e^{(n+i) \alpha},
$$

where $t_{i}\left(\alpha^{\prime}, \alpha^{\prime \prime}, \ldots, \alpha^{(k)}, h, h^{\prime}, \ldots, h^{(k)}\right) \quad(i=0,1,2, \ldots, m)$ are differential polynomials in $\alpha^{\prime}, \alpha^{\prime \prime}, \ldots, \alpha^{(k)}, h, h^{\prime}, \ldots, h^{(k)}$.

We now show that

$$
t_{i}\left(\alpha^{\prime}, \alpha^{\prime \prime}, \ldots, \alpha^{(k)}, h, h^{\prime}, \ldots, h^{(k)}\right) \not \equiv 0,
$$

for $i=0, m$.

On the contrary we suppose that $t_{i} \equiv 0$ for $i=0, m$. Then from (2.38) we have $\left(f^{n+i}\right)^{(k)} \equiv 0$ for $i=0, m$ and so $f$ is a polynomial, which is a contradiction. Hence

$$
t_{i}\left(\alpha^{\prime}, \alpha^{\prime \prime}, \ldots, \alpha^{(k)}, h, h^{\prime}, \ldots, h^{(k)}\right) \not \equiv 0,
$$

for $i=0, m$. Also $(2.36)$ yields $\left[f^{n} P(f)\right]^{(k)} \not \equiv 0$.

From (2.36) and (2.38) we obtain

$$
\bar{N}\left(r, 0 ; t_{m} e^{m \alpha(z)}+\cdots+t_{1} e^{\alpha}+t_{0}\right) \leq N\left(r, 0 ; p^{2}\right)=S(r, f) .
$$

Since $\alpha$ is an entire function, we obtain $T\left(r, \alpha^{(j)}\right)=S(r, f)$ for $j=1,2, \ldots, k$. Hence $T\left(r, t_{i}\right)=S(r, f)$ for $i=0,1,2, \ldots, m$. So from (2.39) and using second fundamental theorem for small functions $\{$ see $[20]\}$, we obtain

$$
\begin{aligned}
m T(r, f)= & T\left(r, t_{m} e^{m \alpha}+\cdots+t_{1} e^{\alpha}\right)+S(r, f) \\
\leq & \bar{N}\left(r, 0 ; t_{m} e^{m \alpha}+\cdots+t_{1} e^{\alpha}\right)+\bar{N}\left(r, \infty ; t_{m} e^{m \alpha}+\cdots+t_{1} e^{\alpha}\right) \\
& +\bar{N}\left(r, 0 ; t_{m} e^{m \alpha}+\cdots+t_{1} e^{\alpha}+t_{0}\right)+(\varepsilon+o(1)) T(r, f) \\
\leq & \bar{N}\left(r, 0 ; t_{m} e^{(m-1) \alpha}+\cdots+t_{1}\right)+(\varepsilon+o(1)) T(r, f) \\
\leq & (m-1) T(r, f)+(\varepsilon+o(1)) T(r, f)
\end{aligned}
$$

for all $\varepsilon>0$. Take $\varepsilon<1$ and we obtain a contradiction. This completes the Lemma.

LEMma 13 [2]. Let $f$ and $g$ be two non-constant meromorphic functions sharing 1 IM. Then

$$
\begin{aligned}
& \bar{N}_{L}(r, 1 ; f)+2 \bar{N}_{L}(r, 1 ; g)+\bar{N}_{E}^{(2}(r, 1 ; f)-\bar{N}_{f>1}(r, 1 ; g)-\bar{N}_{g>1}(r, 1 ; f) \\
& \quad \leq N(r, 1 ; g)-\bar{N}(r, 1 ; g) .
\end{aligned}
$$


Lemma 14 [2]. Let $f, g$ share 1 IM. Then

$$
\bar{N}_{L}(r, 1 ; f) \leq \bar{N}(r, 0 ; f)+\bar{N}(r, \infty ; f)+S(r, f)
$$

Lemma 15 [2]. Let $f, g$ share 1 IM. Then

(i) $\bar{N}_{f>1}(r, 1 ; g) \leq \bar{N}(r, 0 ; f)+\bar{N}(r, \infty ; f)-N_{0}\left(r, 0 ; f^{\prime}\right)+S(r, f)$

(ii) $\bar{N}_{g>1}(r, 1 ; f) \leq \bar{N}(r, 0 ; g)+\bar{N}(r, \infty ; g)-N_{0}\left(r, 0 ; g^{\prime}\right)+S(r, g)$.

LEMma 16. Suppose that $f$ and $g$ be two non-constant meromorphic functions. Let $F=\left[f^{n} P(f)\right]^{(k)}, G=\left[g^{n} P(g)\right]^{(k)}$, where $n(\geq 1), k(\geq 1)$, are integers. If $f, g$ share $\infty I M$ and $V \equiv 0$, then $F \equiv G$.

Proof. Suppose $V \equiv 0$. Then by integration we obtain

$$
1-\frac{1}{F} \equiv A\left(1-\frac{1}{G}\right) .
$$

If $z_{0}$ is a pole of $f$ then it is a pole of $g$. Hence from the definition of $F$ and $G$ we have $\frac{1}{F\left(z_{0}\right)}=0$ and $\frac{1}{G\left(z_{0}\right)}=0$. So $A=1$ and hence $F \equiv G$.

LEMma 17. Suppose that $f$ and $g$ be two non-constant meromorphic functions. $F, G$ be defined as in Lemma 16 and $H \not \equiv 0$. If $f, g$ share $\infty I M$ and $F, G$ share 1 $I M$, then

$$
\begin{aligned}
\left(n+m^{* *}-3 k-3\right) \bar{N}(r, \infty ; f) \leq & 2\left(k+m^{* *}+1\right)\{T(r, f)+T(r, g)\} \\
& +S(r, f)+S(r, g) .
\end{aligned}
$$

Similar result holds for $g$ also.

Proof. Suppose $\infty$ is an e.v.P of $f$ and $g$ then the lemma follows immediately.

Next suppose $\infty$ is not an e.v.P of $f$ and $g$. Since $H \not \equiv 0$ from Lemma 16 we have $V \not \equiv 0$. We suppose that $z_{0}$ is a pole of $f$ with multiplicity $q$ and a pole of $g$ with multiplicity $r$. Clearly $z_{0}$ is a pole of $F$ with multiplicity $\left(n+m^{* *}\right) q+k$ and a pole of $G$ with multiplicity $\left(n+m^{* *}\right) r+k$. Noting that $f, g$ share $\infty$ IM from the definition of $V$ it is clear that $z_{0}$ is a zero of $V$ with multiplicity at least $n+m^{* *}+k-1$. Now using the Milloux theorem \{see [7], p. 55\}, and Lemma 1, we obtain from the definition of $V$ that

$$
m(r, V)=S(r, f)+S(r, g) .
$$


Also by Lemma 14 we get

$$
\begin{aligned}
\bar{N}_{*}(r, 1 ; F, G)= & \bar{N}_{L}(r, 1 ; F)+\bar{N}_{L}(r, 1 ; G) \\
\leq & \bar{N}(r, 0 ; F)+\bar{N}(r, \infty ; F)+N(r, 0 ; G) \\
& +\bar{N}(r, \infty ; G)+S(r, f)+S(r, g) \\
\leq & 2(k+1) \bar{N}(r, \infty, f)+\left(k+m^{*}+1\right) T(r, f) \\
& +\left(k+m^{*}+1\right) T(r, g)+S(r, f)+S(r, g)
\end{aligned}
$$

Thus using Lemmas 1 and 2 we get

$$
\begin{aligned}
\left(n+m^{* *}+k-1\right) \bar{N}(r, \infty ; f) \leq & N(r, 0 ; V) \\
\leq & T(r, V)+O(1) \\
\leq & N(r, \infty ; V)+m(r, V)+O(1) \\
\leq & \bar{N}(r, 0 ; F)+\bar{N}(r, 0 ; G)+\bar{N}_{*}(r, 1 ; F, G) \\
& +S(r, f)+S(r, g) \\
\leq & N_{k+1}\left(r, 0 ; f^{n} P(f)\right)+N_{k+1}\left(r, 0 ; g^{n} P(g)\right) \\
& +k \bar{N}(r, \infty ; f)+k \bar{N}(r, \infty ; g) \\
& +\bar{N}_{*}(r, 1 ; F, G)+S(r, f)+S(r, g) \\
\leq & N_{k+1}\left(r, 0 ; f^{n}\right)+N_{k+1}(r, 0 ; P(f))+N_{K+1}\left(r, 0 ; g^{n}\right) \\
& +N_{k+1}(r, 0 ; P(g))+2 k \bar{N}(r, \infty ; f)+\bar{N}_{*}(r, 1 ; F, G) \\
& +S(r, f)+S(r, g) \\
\leq & (k+1) \bar{N}(r, 0 ; f)+N(r, 0 ; P(f))+(k+1) \bar{N}(r, 0 ; g) \\
& +N(r, 0 ; P(g))+2 k \bar{N}(r, \infty ; f)+\bar{N}_{*}(r, 1 ; F, G) \\
& +S(r, f)+S(r, g) .
\end{aligned}
$$

This gives

$$
\begin{aligned}
\left(n+m^{* *}-3 k-3\right) \bar{N}(r, \infty ; f) \leq & 2\left(k+m^{* *}+1\right)\{T(r, f)+T(r, g)\} \\
& +S(r, f)+S(r, g) .
\end{aligned}
$$

This completes the proof of the lemma. 


\section{Proof of the Theorem}

Proof of Theorem 1. Let $F=\frac{\left[f^{n} P(f)\right]^{(k)}}{p}$ and $G=\frac{\left[g^{n} P(g)\right]^{(k)}}{p}$, where $P(w)=$ $\lambda w^{m}+\mu$. It follows that $F$ and $G$ share 1 IM. Also $f$ and $g$ share $\infty$ IM.

Case 1: Let $H \not \equiv 0$.

From (2.1) it can be easily calculated that the possible poles of $H$ occur at (i) multiple zeros of $F$ and $G$, (ii) those 1 points of $F$ and $G$ whose multiplicities are different, (iii) poles of $f$ and $g$ with different multiplicities, (iv) zeros of $F^{\prime}\left(G^{\prime}\right)$ which are not the zeros of $F(F-1)(G(G-1))$. Since $H$ has only simple poles we get

$$
\begin{aligned}
N(r, \infty ; H) \leq & \bar{N}_{*}(r, \infty ; f, g)+\bar{N}_{*}(r, 1 ; F, G)+\bar{N}(r, 0 ; F \mid \geq 2) \\
& +\bar{N}(r, 0 ; G \mid \geq 2)+\bar{N}_{0}\left(r, 0 ; F^{\prime}\right)+\bar{N}_{0}\left(r, 0 ; G^{\prime}\right)
\end{aligned}
$$

where $\bar{N}_{0}\left(r, 0 ; F^{\prime}\right)$ is the reduced counting function of those zeros of $F^{\prime}$ which are not the zeros of $F(F-1)$ and $\bar{N}_{0}\left(r, 0 ; G^{\prime}\right)$ is similarly defined.

Here we see that

$$
N_{E}^{1)}(r, 1 ; F \mid=1) \leq N(r, 0 ; H) \leq N(r, \infty ; H)+S(r, F)+S(r, G) .
$$

Now using Lemmas 3, 13, 14, 15, (3.1) and (3.2) we get

$$
\begin{aligned}
\bar{N}(r, 1 ; F) \leq & N_{E}^{1)}(r, 1 ; F)+\bar{N}_{L}(r, 1 ; F)+\bar{N}_{L}(r, 1 ; G)+\bar{N}_{E}^{(2}(r, 1 ; F) \\
\leq & \bar{N}(r, \infty ; f)+\bar{N}(r, 0 ; F \mid \geq 2)+\bar{N}(r, 0 ; G \mid \geq 2)+\bar{N}_{*}(r, 1 ; F, G) \\
& +\bar{N}_{L}(r, 1 ; F)+\bar{N}_{L}(r, 1 ; G)+\bar{N}_{E}^{(2}(r, 1 ; F)+\bar{N}_{0}\left(r, 0 ; F^{\prime}\right) \\
& +\bar{N}_{0}\left(r, 0 ; G^{\prime}\right)+S(r, f)+S(r, g) \\
\leq & \bar{N}(r, \infty ; f)+\bar{N}(r, 0 ; F \mid \geq 2)+\bar{N}(r, 0 ; G \mid \geq 2) \\
& +2 \bar{N}_{L}(r, 1 ; F)+2 \bar{N}_{L}(r, 1 ; G)+\bar{N}_{E}^{(2}(r, 1 ; F) \\
& +\bar{N}_{0}\left(r, 0 ; F^{\prime}\right)+\bar{N}_{0}\left(r, 0 ; G^{\prime}\right)+S(r, f)+S(r, g) \\
\leq & \bar{N}(r, \infty ; f)+\bar{N}(r, 0 ; F \mid \geq 2)+\bar{N}(r, 0 ; G \mid \geq 2)+\bar{N}_{F>1}(r, 1 ; G) \\
& +\bar{N}_{G>1}(r, 1 ; F)+\bar{N}_{L}(r, 1 ; F)+N(r, 1 ; G)-\bar{N}(r, 1 ; G) \\
& +\bar{N}_{0}\left(r, 0 ; F^{\prime}\right)+\bar{N}_{0}\left(r, 0 ; G^{\prime}\right)+S(r, f)+S(r, g)
\end{aligned}
$$




$$
\begin{aligned}
\leq & 4 \bar{N}(r, \infty ; f)+N_{2}(r, 0 ; F)+\bar{N}(r, 0 ; F)+N_{2}(r, 0 ; G)+N(r, 1 ; G) \\
& -\bar{N}(r, 1 ; G)+\bar{N}_{0}\left(r, 0 ; G^{\prime}\right)+\bar{N}_{0}\left(r, 0 ; F^{\prime}\right)+S(r, f)+S(r, g) \\
\leq & 4 \bar{N}(r, \infty ; f)+N_{2}(r, 0 ; F)+\bar{N}(r, 0 ; F)+N_{2}(r, 0 ; G) \\
& +N\left(r, 0 ; G^{\prime} \mid G \neq 0\right)+\bar{N}_{0}\left(r, 0 ; F^{\prime}\right)+S(r, f)+S(r, g) \\
\leq & 5 \bar{N}(r, \infty ; f)+N_{2}(r, 0 ; F)+\bar{N}(r, 0 ; F)+N_{2}(r, 0 ; G) \\
& +\bar{N}(r, 0 ; G)+\bar{N}_{0}\left(r, 0 ; F^{\prime}\right)+S(r, f)+S(r, g) .
\end{aligned}
$$

Hence using (3.3), Lemmas 1, 2 and 17 we get from second fundamental theorem that

$$
\begin{aligned}
& \left(n+m^{*}\right) T(r, f) \leq \bar{N}(r, 0 ; F)+\bar{N}(r, \infty ; F)+\bar{N}(r, 1 ; F) \\
& +N_{k+2}\left(r, 0 ; f^{n} P(f)\right)-N_{2}(r, 0 ; F)-N_{0}\left(r, 0 ; F^{\prime}\right) \\
& \leq 6 \bar{N}(r, \infty, f)+N_{2}(r, 0 ; F)+2 \bar{N}(r, 0 ; F) \\
& +N_{k+2}\left(r, 0 ; f^{n} P(f)\right)+N_{2}(r, 0 ; G) \\
& +\bar{N}(r, 0 ; G)-N_{2}(r, 0 ; F)+S(r, f)+S(r, g) \\
& \leq 6 \bar{N}(r, \infty ; f)+N_{k+2}\left(r, 0 ; f^{n} P(f)\right)+2 \bar{N}(r, 0 ; F) \\
& +N_{2}(r, 0 ; G)+\bar{N}(r, 0 ; G)+S(r, f)+S(r, g) \\
& \leq 6 \bar{N}(r, \infty ; f)+N_{k+2}\left(r, 0 ; f^{n} P(f)\right)+2 k \bar{N}(r, \infty ; f) \\
& +2 N_{k+1}\left(r, 0 ; f^{n} P(f)\right)+k \bar{N}(r, \infty ; g)+N_{k+2}\left(r, 0 ; g^{n} P(g)\right) \\
& +k \bar{N}(r, \infty ; g)+N_{k+1}\left(r, 0 ; g^{n} P(g)\right)+S(r, f)+S(r, g) \\
& \leq(4 k+6) \bar{N}(r, \infty ; f)+(3 k+4) \bar{N}(r, 0 ; f)+3 T(r, P(f)) \\
& +(2 k+3) \bar{N}(r, 0 ; g)+2 T(r, P(g))+S(r, f)+S(r, g) \\
& \leq \frac{12\left(k+m^{* *}+1\right)}{n+m^{* *}-3 k-3}\{T(r, f)+T(r, g)\}+4 k T(r, f) \\
& +(3 k+4) \bar{N}(r, 0 ; f)+3 T(r, P(f)) \\
& +(2 k+3) \bar{N}(r, 0 ; g)+2 T(r, P(g))+S(r, f)+S(r, g) \\
& \leq\left\{9 k+5 m^{*}+7+\frac{24\left(k+m^{*}+1\right)}{n+m^{*}-3 k-3}\right\} T(r)+S(r) .
\end{aligned}
$$


In a similar way we can obtain

$$
\left(n+m^{*}\right) T(r, g) \leq\left\{9 k+5 m^{*}+7+\frac{24\left(k+m^{*}+1\right)}{n+m^{*}-3 k-3}\right\} T(r)+S(r) .
$$

Combining (3.4) and (3.5) we see that

$$
\left[\frac{\left(n-9 k-4 m^{*}-7\right)\left(n+m^{*}-3 k-3\right)-24\left(k+m^{*}+1\right)}{n+m^{*}-3 k-3}\right] T(r) \leq S(r) .
$$

When $n>9 k+4 m^{*}+11$, (3.6) leads to a contradiction.

Case 2: Let $H \equiv 0$. Then by Lemma 9, we get either

$$
f^{n}\left(\lambda f^{m}+\mu\right) \equiv g^{n}\left(\lambda g^{m}+\mu\right)
$$

or

$$
\left[f^{n}\left(\lambda f^{m}+\mu\right)\right]^{(k)}\left[g^{n}\left(\lambda g^{m}+\mu\right)\right]^{(k)} \equiv p^{2}
$$

We now consider the following two subcases:

Subcase 2.1: Let $\lambda \mu \neq 0$. By Lemma 12 we must have

$$
\left[f^{n}\left(\lambda f^{m}+\mu\right)\right]^{(k)}\left[g^{n}\left(\lambda g^{m}+\mu\right)\right]^{(k)} \not \equiv p^{2}
$$

Next we consider the relation (3.7). Let $m=1$. In this case noting that $d=1=$ $(1, n)$, proceeding in the same way as done in Lemma 6 of [11] we can show when $\Theta(\infty, f)+\Theta(\infty, g)>4 / n$, we have $f \equiv g$.

Next we suppose $m \geq 2$. Let $f \not \equiv t g$ for a constant $t$ satisfying $t^{d}=1$, where $d=\operatorname{gcd}(n, m)$. We put $h=\frac{f}{g}$. Then $h^{d} \not \equiv 1$, i.e., $\left(h-v_{0}\right)\left(h-v_{1}\right) \cdots\left(h-v_{d-1}\right) \not \equiv 0$, where $v_{k}=\exp \left(\frac{2 k \pi i}{d}\right), k=0,1,2, \ldots, d-1$. First suppose that $h$ is constant. Now (3.7) implies

$$
\mu g^{m}\left(h^{n+m}-1\right) \equiv-\lambda\left(h^{n}-1\right) .
$$

Since $\operatorname{gcd}(n, m)=d$, it follows that $\operatorname{gcd}(n+m, n)=d$. Eliminating $d$ common factors namely $h-v_{k}, k=0,1, \ldots, d-1$ from both sides we are left with

$$
a g^{m}\left(h-\alpha_{1}\right)\left(h-\alpha_{2}\right) \cdots\left(h-\alpha_{n+m-d}\right) \equiv\left(h-\beta_{1}\right)\left(h-\beta_{2}\right) \cdots\left(h-\beta_{n-d}\right),
$$


where $\alpha_{i}$ and $\beta_{j}$ are those zeros of $h^{n+m}-1$ and $h^{n}-1$ which are not the zeros of $h^{d}-1, i=1,2, \ldots, n+m-d$ and $j=1,2, \ldots, n-d$. Also we note that none of the $\alpha_{i}$ 's coincides with $\beta_{j}$ 's. So if $h=\alpha_{i}$ or $\beta_{j}$, then we have either $\left(h-\beta_{1}\right)\left(h-\beta_{1}\right) \cdots\left(h-\beta_{n-1-d}\right) \equiv 0$ or $g \equiv 0$ and in both case we get contradictions. So we assume neither $h^{n+m} \equiv 1$ nor $h^{n} \equiv 1$. Hence we may write

$$
g^{m}=-\frac{\lambda}{\mu} \frac{h^{n}-1}{h^{n+m}-1} .
$$

It follows from above that $g$ is a constant, which is impossible. So $h$ is nonconstant. We observe that since a non-constant meromorphic function can not have more than two Picard exceptional values $h$ can take at least $n+m-d-2$ values among $u_{j}=\exp \left(\frac{2 j \pi i}{n+m}\right)$, where $j=0,1,2, \ldots, n+m-1$. Since $g^{m}$ has no simple pole, and so $h-u_{j}$ has no simple zero for at least $n+m-d-2$ values of $u_{j}$, for $j=0,1,2, \ldots, n+m-1$ and for these values of $j$ we have $\Theta\left(u_{j} ; h\right) \geq \frac{1}{2}$, which leads to a contradiction.

Therefore $h^{d} \equiv 1$. i.e., $f \equiv t g$ for a constant $t$ satisfying $t^{d}=1$, where $d=\operatorname{gcd}(n, m)$.

Subcase 2.2: Let $\lambda \mu=0$ but $|\lambda|+|\mu| \neq 0$. Then from (3.7) we get $f^{n+m^{*}} \equiv g^{n+m^{*}}$ and so $f \equiv t g$, where $t$ is a constant satisfying $t^{n+m^{*}}=1$.

Also from (3.8) we get either

$$
\lambda^{2}\left[f^{n+m}\right]^{(k)}\left[g^{n+m}\right]^{(k)} \equiv p^{2}
$$

or

$$
\mu^{2}\left[f^{n}\right]^{(k)}\left[g^{n}\right]^{(k)} \equiv p^{2}
$$

Then by Lemma 11, we get if $p(z)$ is not a constant then $f=c_{1} e^{c Q(z)}, g=$ $c_{2} e^{-c Q(z)}$, where $Q(z)=\int_{0}^{z} p(z) d z$ and $c_{1}, c_{2}$ and $c$ are three constants satisfying either $\mu^{2}(n c)^{2}\left(c_{1} c_{2}\right)^{n}=-1$ or $\lambda^{2}[(n+m) c]^{2}\left(c_{1} c_{2}\right)^{n+m}=-1$, if $p(z)$ is a nonzero constant $b$, then $f=c_{3} e^{d z}, g=c_{4} e^{-d z}$, where $c_{3}, c_{4}$ and $d$ are three constants satisfying either $(-1)^{k} \lambda^{2}\left(c_{1} c_{2}\right)^{n+m}[(n+m) c]^{2 k}=b^{2}$ or $(-1)^{k} \mu^{2}\left(c_{1} c_{2}\right)^{n}[n c]^{2 k}=b^{2}$. This completes the proof.

Proof of Theorem 2. Let $F=\left[f^{n} P(f)\right]^{(k)} / p(z)$ and $G=\left[g^{n} P(g)\right]^{(k)} / p(z)$. It follows that $F$ and $G$ share 1 IM and $f, g$ share $\infty$ IM. We omit the proof since the proof of the theorem can be carried out in the line of proof of Theorem 1. 


\section{Acknowledgement}

The authors are grateful to the referee for his/her valuable comments and suggestions to-wards the improvement of the paper.

This research work is supported by the Council Of Scientific and Industrial Research, Extramural Research Division, CSIR Complex, Pusa, New Delhi-110012, India, under the sanction project no. 25(0229)/14/EMR-II.

\section{References}

[ 1 ] T. C. Alzahary and H. X. Yi, Weighted value sharing and a question of I. Lahiri, Complex Var. Theory Appl. 49(15) (2004), 1063-1078.

[2] A. Banerjee, Meromorphic functions sharing one value, Int. J. Math. Sci. 22 (2005), 3587-3598.

[ 3 ] J. Dou, X. G. Qi and L. Z. Yang, Entire functions that share fixed points, Bull. Malays. Math., 34(2) (2011), 355-367.

[4] M. L. Fang and X. H. Hua, Entire functions that share one value, J. Nanjing Univ. Math. Biquarterly, 13(1) (1996), 44-48.

[ 5 ] M. L. Fang, Uniqueness and value-sharing of entire functions, Comput. Math. Appl., 44 (2002), 823-831.

[6] G. Frank, Eine Vermutung Von Hayman über Nullslellen meromorphic Funktion, Math. Z., 149 (1976), 29-36.

[ 7 ] W. K. Hayman, Meromorphic Functions, The Clarendon Press, Oxford (1964).

[ 8 ] W. K. Hayman, Picard values of meromorphic functions and their derivatives, Annal. Math., Second Series, 70(1) (Jul., 1959), pp. 9-42.

[9] I. Lahiri, Weighted sharing and uniqueness of meromorphic functions, Nagoya Math. J., 161 (2001), 193-206.

[10] I. Lahiri, Weighted value sharing and uniqueness of meromorphic functions, Complex Var. Theory Appl., 46 (2001), 241-253.

[11] I. Lahiri, On a question of Hong Xun Yi, Arch. Math. (Brno), 38 (2002), 119-128.

[12] I. Lahiri and S. Dewan, Value distribution of the product of a meromorphic function and its derivative, Kodai Math. J., 26 (2003), 95-100.

[13] I. Lahiri and A. Sarkar, Nonlinear differential polynomials sharing 1-points with weight two, Chinese J. Contemp. Math., 25(3) (2004), 325-334.

[14] Y. Liu and L. Z. Yang, Some Further Results On Uniqueness Of Entire Functions And Fixed Points, Kyungpook Math. J, 53 (2013), 371-383.

[15] X. G. Qi and L. Z. Yang, Uniqueness of entire functions and fixed points, An. Polon. Math., 97 (2010), 87-100.

[16] P. Sahoo, Meromorphic functions that share fixed points with finite weights, Bull. Math. Anal. Appl., 2 (2010), 106-118.

[17] J. Wang, W. Lu and Y. Chen, Value sharing of meromorphic functions and their derivatives, Applied Math. E-Notes, 11 (2011), 91-100.

[18] C. C. Yang, On deficiencies of differential polynomials II, Math. Z. Vol. 125 (1972), $107-112$.

[19] C. C. Yang and X. H. Hua, Uniqueness and value sharing of meromorphic functions, Ann. Acad. Sci. Fenn. Math. 22 (1997), 395-406.

[20] K. Yamanoi, The second main theorem for small functions and related problems, Acta Math. 192 (2004), 225-294.

[21] H. X. Yi, On characteristic function of a meromorphic function and its derivative, Indian J. Math. 33(2) (1991), 119-133. 
[22] C. C. Yang and H. X. Yi, Uniqueness Theory of Meromorphic Functions, Kluwer Academic Publishers, Dordrecht/Boston/London, 2003.

[23] Q. C. Zhang, Meromorphic function that shares one small function with its derivative, J. Inequal. Pure Appl. Math., 6(4) (2005), Art. 116 [ONLINE http://jipam.vu.edu.au/].

[24] X. Y. Zhang and W. C. Lin, Uniqueness and value sharing of entire functions, J. Math. Anal. Appl., 343 (2008), 938-950.

\author{
Abhijit Banerjee \\ Department of Mathematics \\ University of Kalyani \\ West Bengal 741235 \\ India \\ E-mail: abanerjee_kal@yahoo.co.in \\ abanerjee_kal@rediffmail.com \\ Sujoy Majumder \\ Department of Mathematics \\ Katwa College, Katwa \\ West Bengal-713130 \\ India \\ E-mail: sujoy.katwa@gmail.com
}

